University of Nebraska - Lincoln

DigitalCommons@University of Nebraska - Lincoln

Faculty Publications: Department of Entomology

Entomology, Department of

2005

Quality of Transgenic Laboratory Strains of Cochliomyia

hominivorax (Diptera: Calliphoridae)

Margaret L. Allen

USDAĐARS, Midwest Livestock Insects Research Unit

Philip J. Scholl

USDAEARS, Midwest Livestock Insects Research Unit

Follow this and additional works at: https://digitalcommons.unl.edu/entomologyfacpub

Part of the Entomology Commons

Allen, Margaret L. and Scholl, Philip J., "Quality of Transgenic Laboratory Strains of Cochliomyia hominivorax (Diptera: Calliphoridae)" (2005). Faculty Publications: Department of Entomology. 209.

https://digitalcommons.unl.edu/entomologyfacpub/209

This Article is brought to you for free and open access by the Entomology, Department of at DigitalCommons@University of Nebraska - Lincoln. It has been accepted for inclusion in Faculty Publications: Department of Entomology by an authorized administrator of DigitalCommons@University of Nebraska - Lincoln. 


\title{
Quality of Transgenic Laboratory Strains of Cochliomyia hominivorax (Diptera: Calliphoridae)
}

\author{
MARGARET L. ALLEN ${ }^{1}$ AND PHILIP J. SCHOLL \\ USDA-ARS, Midwest Livestock Insects Research Unit, 305 Plant Industry Building, University of Nebraska-East Campus,
} Lincoln, NE 68583-0938

\begin{abstract}
J. Econ. Entomol. 98(6): 2301-2306 (2005)
ABSTRACT Genetically modified, mass reared insects present novel possibilities for the future of insect control. One concern about manipulation of insects is a possible loss of strain quality due to the introduction of a foreign gene of any sort into the insect genome. Eight transgenic strains of screwworm, Cochliomyia hominivorax (Coquerel) (Diptera: Calliphoridae), were compared with the wild-type parental laboratory strain in laboratory culture. Measurements of average fertility, fecundity, larval productivity, and longevity were analyzed. Two transgenic strains had significantly lower larval productivity than controls, one of which was explained by a homozygous lethal insertion of the transgene. Another strain produced significantly fewer eggs than controls. Overall strain characteristics, including measurements from egg, larva, pupa, and adult stages, were compared. Transgenic colonies did not consistently show significantly lower individual or aggregate strain quality characteristics than the control parental colony; hence, the presence of the transgene used to produce the strains tested did not incur a discrete cost to the colonies of laboratory-reared C. hominivorax.
\end{abstract}

KEY WORDS Cochliomyia hominivorax, mass rearing, quality control, transgenic insect, sterile insect technique

TRANSFORMATION OF PEST INSECTS may be used to improve current sterile insect technique (SIT) programs (Handler 2002). SIT programs involve the mass release of laboratory-reared, sterilized insects into the environment. Some key issues involved in mass release of sterile insects relate to strain quality of released insects, including the economical rearing of mass quantities of robust insects (Knipling 1955). Transgenic insects must be capable of surviving, mating, and reproducing efficiently in culture to be used successfully in mass production. Furthermore, the mass rearing fitness of the transgenic strain must be equivalent to the current production strain if it is to be effective. Therefore, it is critical to analyze strain characteristics of transgenic insects, including fertility, fecundity, and larval health.

Through state and international cooperative efforts with the U.S. Department of Agriculture, the screwworm, Cochliomyia hominivorax (Coquerel) (Diptera: Calliphoridae), was eradicated from the island of $\mathrm{Cu}-$ racao in 1954, the southeastern United States in 1959, and subsequently in the remaining United States, Mex-

Mention of trade names or commercial products in this article is solely for the purpose of providing specific information and does not imply recommendation or endorsement by the USDA. The USDA is an equal opportunity/affirmative action employer, and all agency services are available without discrimination.

${ }^{1}$ Current address: Mid-South Area, Biological Control of Pests Research Unit, Stoneville MS 38776. ico, Central America, and Libya (Wyss 2000). The screwworm remains endemic in parts of South America and the Caribbean. The eradication zone has progressed to the Darien Gap in Panama, where flies currently are released to prevent reinvasion of the northern continent. At present, flies are sterilized using ionizing radiation at a mass rearing production facility in Tuxtla-Gutierrez, Chiapas, Mexico.

Several strains of transgenic C. hominivorax incorporating an enhanced green fluorescent protein (EGFP) marker have been established as stable colonies (Allen et al. 2004b). Each stable strain had at least a single copy of the transgene incorporated into a distinctive genomic location. These strains were used to assess postlarval fitness costs associated with genomic transgene integration, including average weight of pupae, adult emergence, male ratio, and mating competitiveness (Allen et al. 2004a). The strain characteristics associated with fecundity, fertility, larval productivity, and longevity are presented, along with an unweighted aggregated strain quality summary.

\section{Materials and Methods}

Insect Stocks. All transgenic C. hominivorax colonies were derived from the P95 strain, the most current strain used in the SIT program that was established in 1995 from insects collected in Panama. Another non- 
transgenic strain, CR91, was established in 1991 from insects collected in Costa Rica and was used for a short time as the SIT strain. Insects were reared according to USDA-ARS and Animal and Plant Health Inspection Service (APHIS) established protocols (Melvin and Bushland 1936, Taylor et al. 1991).

Although insect rearing at the C. hominivorax Biosecure Facility in Lincoln, NE, was performed using established protocols, occasional fluctuations in rearing environment occurred. However, each cohort of insects was processed as a group and exposed to the same fluctuations, because they were in the same incubators and were handled by the same technicians. Transgenic colonies of $C$. hominivorax required some modified procedures. Two differences between the handling of the transgenic and parental strains are notable. First, verification of transgenic status was through visualization of the fluorescent protein marker (pUbnlsEGFP), so each strain was screened by examination using fluorescence illumination before pupation. The pupal and imaginal cuticles are completely opaque, making screening of these stages impossible without destruction of the insect. For each transgenic strain, fully mature ("crawler") third instars were separated from rearing medium and examined each generation. This procedure usually was performed on one day (day 5) during the development of each cohort of insects. Unscreened pupae were discarded. Standard rearing practices for nontransgenic strains allowed insects to pupate unmolested for several days, with pupae collected on day 9 or 10 . Therefore, a much smaller portion of pupae was collected for each generation from the transgenic colonies, and a smaller number of transgenic adults were available to produce eggs. The second difference between standard and transgenic colonies was that the screening process of transgenic insects was nonrandom because smaller larvae were discarded in favor of larger specimens. To provide a control for these handling differences between transgenic and nontransgenic parental colonies, a subculture colony of wild-type P95 insects was established (Allen et al. 2004a) and used in the comparison of fertility. This colony was screened (mock-screened, because there were no fluorescent specimens selected) in the same manner as the transgenic colonies.

Strain Quality Measurements. Parameters measured on a continuous basis included total egg production, egg hatch, average and total pupal weight, adult emergence, and male:female ratio. These measurements were used to evaluate the overall health and quality of each colony or strain. Once transgenic C. hominivorax strains were established as stable colonies (minimum five generations), strain quality measurements were recorded from these colonies along with the other wild-type and mutant strains of $C$. hominivorax.

Fertility was measured by taking random samples of 95-105 eggs and incubating them for $24 \mathrm{~h}$ at $37^{\circ} \mathrm{C}$ on moist filter paper. Samples were frozen to destroy viable larvae and counted as follows: empty eggs were classified as hatched, eggs containing visibly devel- oped larvae were classified as embryonated, and eggs with undifferentiated contents were classified as unembryonated.

Fecundity was measured by isolating equal numbers of females from wild-type and transgenic colony cages $24 \mathrm{~h}$ before egg collection, and concurrently collecting eggs from all samples. Adults were anesthetized by exposure to carbon dioxide gas. Eightyseven to 100 females were used for the analyses to avoid exclusion of access to the oviposition substrate (ground beef) by overcrowding. The total eggs produced were weighed and the average egg production per female was calculated for each strain.

Larval production was measured by taking a sample of eggs (between 99 and $101 \mathrm{mg}$ ) from each strain and rearing the larvae to maturity. Mature larvae exited the rearing medium and were collected after pupation. No transgenic screening with fluorescence illumination was used. Total weights of the pupae produced by each strain were measured and 100 pupae were weighed to obtain an average pupal weight. The total pupal weight per initial weight of eggs was calculated for each sample, along with the estimated total number of pupae based on average pupal weight.

Longevity was measured by collecting 50 adults from each strain and counting dead individuals daily until all adults were dead and then calculating a mean life span. Adult food (Chaudhury et al. 2000) and water were provided ad libitum.

Statistical Analysis. The measurements of fertility, fecundity, larval production, and longevity were analyzed with the PROC MIXED procedure (SAS Institute 1999), which compares least squares means. A probability of $<0.05$ was assigned as statistically significant. Because the criteria for measured effects were defined as those indicating decrease of strain quality, only statistically significant reductions in quality are presented as significant. The MIXED procedure accommodated the unequal sampling of colonies, sequential sampling of laboratory cultures, and provided for separation of evaluation of fixed effects based on date and strain. The table combining life stage quality calculations (Table 6 ) is not presented as a statistically accurate model but merely for broad illustration.

\section{Results and Discussion}

Based on observations of the transgenic colonies and measurements of postlarval stages (Allen et al. 2004a), strain quality characteristics similar to the parental wild-type strain P95 were anticipated. All gross visual characteristics of the flies at all stages seemed identical; colonies were robust and vigorous. The transgene insert pUbnlsEGFP encoded only the marker, enhanced green fluorescent protein, which was expected to have no physiological effect. Therefore, expected results would confirm a hypothesis that the transgenic element insertion did not incur a cost to the colonies, except as associated with insertion site.

To evaluate the nonrandom handling impact incurred by fluorescent screening, two wild-type con- 
Table 1. Hatch and embryonation rates for unscreened and mock-screened wild-type strains of C. hominivorax

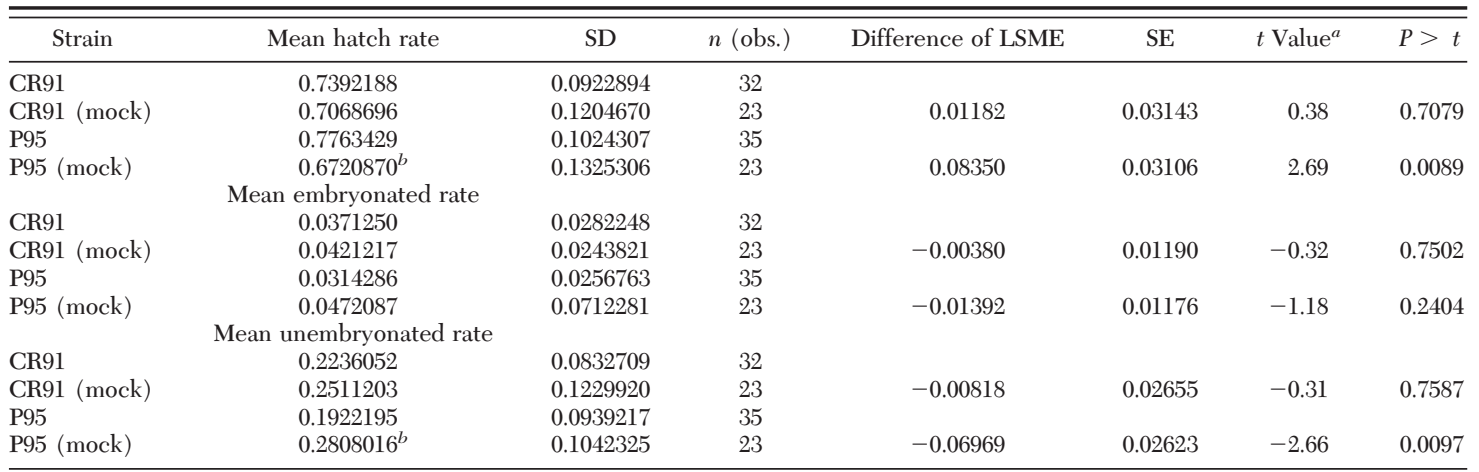

Mock, mock-screened.

${ }^{a} \mathrm{df}=72$.

${ }^{b}$ Statistically significant decrease in strain quality.

trol strains were compared, as in a previous study (Allen et al. 2004a). Cultures of wild-type strains P95 and CR91 that were treated with mock-screening procedures are shown in Table 1. Although CR91 flies did not differ significantly in hatched, embryonated, or unembryonated samples rated, the P95 culture exposed to the screening process showed a significantly lower hatch rate $(t=2.69, \mathrm{df}=72, P=0.0089)$, with corresponding increases in embryonated (not significant) and unembryonated $(t=-2.66, \mathrm{df}=72, P=$ $0.0097)$ rates. The fixed effects for hatch were not correlated to date $(F=1.29 ; \mathrm{df}=37,72 ; P=0.1779)$, nor were they significantly correlated to strain $(F=$ 2.46; df $=3,72 ; P=0.0697)$. The fixed effects for unembryonated also were not correlated to strain $(F=2.41 ; \mathrm{df}=3,72 ; P=0.0740)$ but were significantly correlated to date $(F=1.77 ; \mathrm{df}=37,72 ; P=0.0196)$.

Transgenic strains did not show significantly decreased hatch rate, increased embryonated unhatched or increased unembryonated unhatched rates (Table 2) compared with the mock-screened P95 controls.

Fecundity of one transgenic strain, FOLY, was decreased significantly $(t=-2.29, \mathrm{df}=24, P=0.0311)$ compared with the P95 parental strain (Table 3). The

Table 2. Hatch and embryonation rates for transgenic strains of $C$. hominivorax

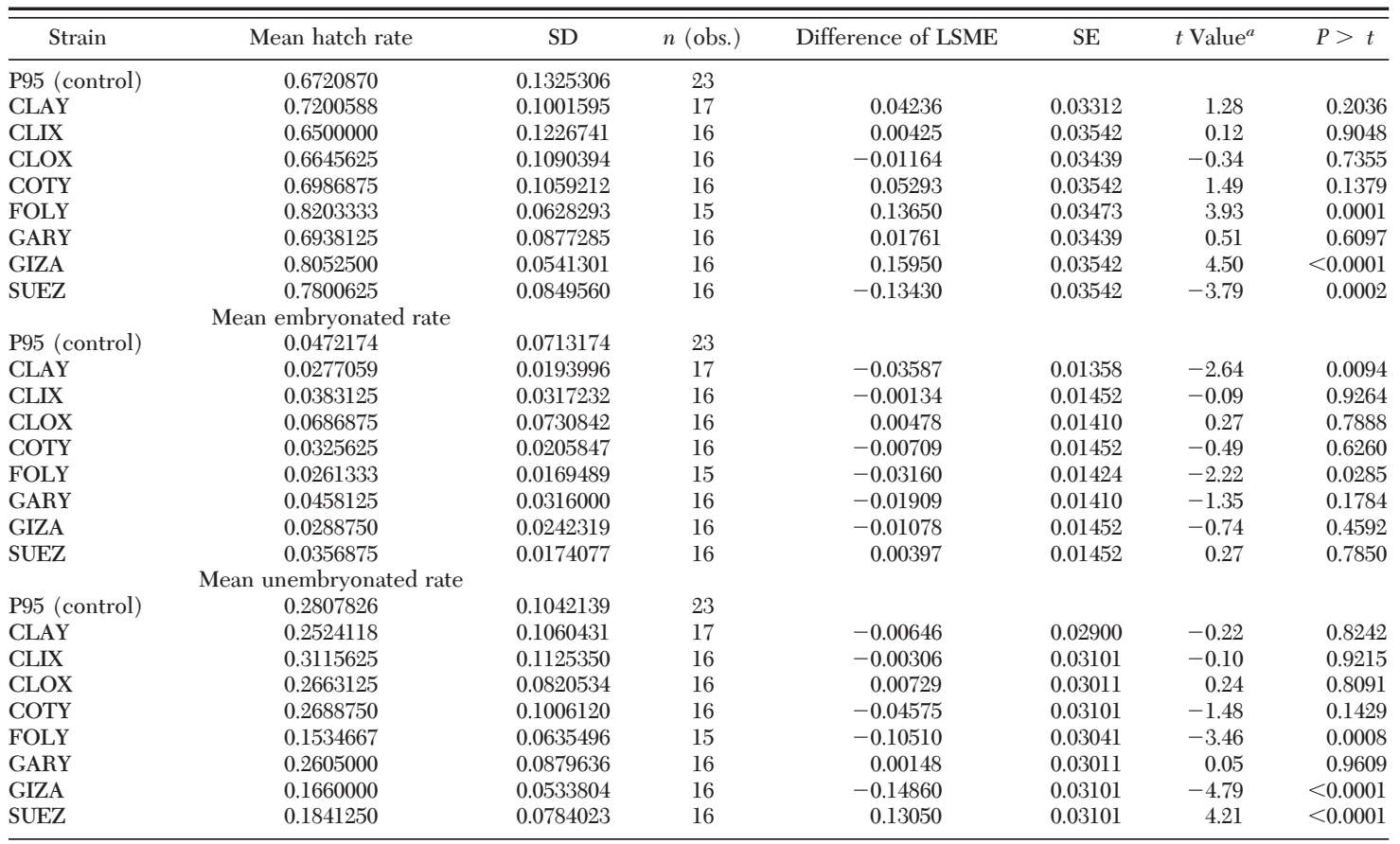

Statistically significant decrease in strain quality.

${ }^{a} \mathrm{df}=111$. 
Table 3. Fecundity of strains of $C$. hominivorax

\begin{tabular}{lccrr}
\hline \hline Strain & Mean fecundity eggs $(\mathrm{mg}) /$ female & SD & Difference of LSME & $t$ Value $^{a}$ \\
\hline P95 & 3.533 & 1.377 & & \\
CLAY & 4.707 & 1.445 & 1.2406 & 2.54 \\
CLIX & 4.821 & 0.769 & 1.4416 & 2.96 \\
CLOX & 4.654 & 0.634 & 1.2991 & 0.0179 \\
COTY & 4.368 & 0.398 & 0.9059 & 0.0069 \\
FOLY & $2.359^{b}$ & 0.663 & 0.0136 \\
GARY & 2.802 & 0.870 & -1.1175 & 0.6411 \\
GIZA & 4.072 & 1.083 & -2.29 \\
SUEZ & 3.854 & 0.632 & 0.7330 & -1.31 \\
\hline
\end{tabular}

${ }^{a} n=4, \mathrm{df}=24, \mathrm{SE}=0.4879$.

${ }^{b}$ Statistically significant decrease compared with P95 control.

fixed effects of both strain $(F=6.83 ; \mathrm{df}=8,24 ; P=$ $0.0001)$ and date $(F=8.60 ; \mathrm{df}=3,24 ; P=0.0005)$ were significant according to least squares means estimate (LSME) comparisons.

Larval productivity, as shown in Table 4, was significantly decreased in the CLIX strain, as measured by both biomass $(t=-2.21, \mathrm{df}=39, P=0.0332)$ and number of larvae $(t=-3.03, \mathrm{df}=39, P=0.0043)$, indicating that the transgene incurred some cost to this strain. There was also a significant decrease in larval number for the GIZA strain $(t=-2.10, \mathrm{df}=39$, $P=0.0421$ ) with no correspondingly significant decrease in biomass. The single transgene insertion of this strain was shown to interrupt to a vital gene (Allen et al. 2004b) that resulted in mortality of homozygotes. Thus, a decrease in larval productivity for this strain was expected, because a heterozygous cross of these individuals produced 25\% homozygous (dead) offspring.

Longevity of fed, mixed sex transgenic strain samples, shown in Table 5, did not show significant reduction compared with P95 controls. Fixed effects were attributed to strain $(F=2.99$; df $=8,16 ; P=$ $0.0295)$ but not date $(F=0.56 ; \mathrm{df}=2,16 ; P=0.5835)$ according to the LSME comparisons.
As a conservative estimate of overall strain quality decreases produced through insect transformation, all measurements less than the control, significant or not, were factored equally (multiplied) together in Table 6. The larval productivity factor was based on the calculated number (rather than biomass) of pupae because this measurement reflected the expected decrease for the lethal insertion strain, GIZA. This table illustrates that, if a conservative cutoff limit for overall comparative quality at 0.80 is set, about half of the strains might qualify as candidates for production strain replacements, whereas half would clearly be inadequate in quality. The 0.80 cutoff, although entirely arbitrary, corresponds roughly with those strains found to be decreased significantly in one or more quality factors. The GARY strain is interesting because although no measured life history characteristic for this strain varied significantly from control, combined absolute measurements gave the appearance of a quality loss.

Several strains showed what seemed to be higher fitness measurements compared with the control, but these apparent increases cannot be attributed to the transgene. The transgene product EGFP is routinely incorporated into microorganisms, plants, insects, and

Table 4. Larval productivity of strains of $C$. hominivorax

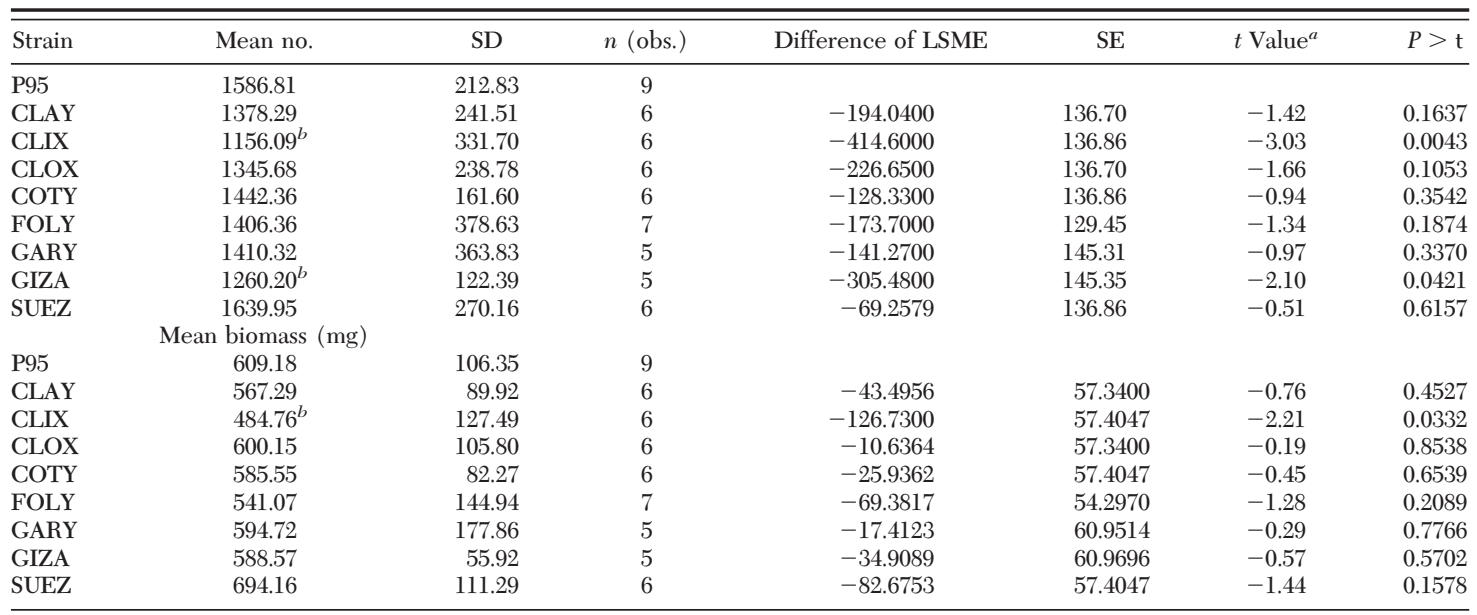

${ }^{a} \mathrm{df}=39$.

${ }^{b}$ Significantly different (decreased) from P95 control $(P<0.05)$. 
Table 5. Adult longevity of transgenic strains of $C$. hominivorax

\begin{tabular}{|c|c|c|c|c|c|}
\hline Strain & Mean longevity adult-days & SD & Difference of LSME & $t$ Value $^{a}$ & $P>\mathrm{t}$ \\
\hline CLAY & 23.4 & 2.3 & 3.4600 & 2.2 & 0.0425 \\
\hline CLOX & 20.6 & 1.3 & 0.7067 & 0.45 & 0.6586 \\
\hline COTY & 19.2 & 3.0 & -0.0733 & -0.47 & 0.6466 \\
\hline FOLY & 16.6 & 1.8 & -3.2933 & -2.1 & 0.0521 \\
\hline GARY & 18.2 & 1.1 & -1.7010 & -1.08 & 0.2946 \\
\hline
\end{tabular}

Statistically significant decrease compared with P95 control.

${ }^{a} n$ (obs.) $=3, \mathrm{df}=16, \mathrm{SE}=1.1099$

mammals as a neutral marker. The genomic site of transgene insertion could be neutral or could disrupt gene function, which should incur a fitness cost to the organism. Although not outside of the realm of speculation, gene disruption cannot be assumed to produce probiotic effects. Therefore, increased fitness measurements are assumed to be the result of selection. Generation of the transgenic strains forced the insects through a selective bottleneck.

The series of experiments comparing overall life history measurements between transgenic and parental strain (P95) wild-type insects indicated that there was no consistent loss of fitness throughout the strains of transgenic C. hominivorax. Specific strains showed some decrease in fecundity, larval productivity, and adult longevity. Because the characteristics were not consistent, existence of the transgene cannot be concluded to affect the quality of the strain. If a neutral transformation does not affect insect quality, transgenic insects that are significantly less fit than wildtype, mass-reared counterparts may be assumed to have problems associated with the genetic insertion site or transgene effect(s).

Because nondrosophilid insect transformation is a relatively recent technology (Handler 2001), few transgenic insects have been evaluated for quality control or fitness characteristics. One fitness study using transgenic insects evaluated the persistence of transgenic Anopheles stephansi (Liston) mosquitoes over several generations. Whereas it was stated that longevity, feeding rate, larval developmental time, and egg-laying rate compared favorably with nontransgenics, population studies showed a decline of frequency and loss of the transgene allele over eight or fewer generations (Catteruccia et al. 2003). This type of study would be inappropriate for evaluating C. hominivorax or other insects for SIT performance, but it would be valuable for environmental risk assessment. Transgenic C. hominivorax will not be designed to replace an endemic population, but rather to eradicate the population by release of mass quantities of sterile individuals that mate and die. For this purpose, an insect that can be mass reared is essential, and the results presented here are supportive to that end.

For practical purposes, multiple strains for any transgenic insect application should be produced, and strain (s) chosen for best overall characteristics should progress to mass culture and field release. A basic premise behind the rationale for using transgenics in SIT is the improvement of the released strain compared with an irradiated insect. Although our studies show favorable indications for mass culture, the goal of producing a genetically sterile strain of C. hominivorax has not been met. Genetically manipulated insects designed to be released without an irradiation step will need to be proven effectively sterilized before consideration of release. Sufficient sterilization must be evaluated based on standards for the pest; $C$. hominivorax is a sufficiently dangerous pest to require diminishingly low tolerance for fertile releases. After sterility is achieved, strain evaluation should progress to application-oriented parameters, including flight ability, and, most importantly, mating competition.

Previous studies evaluated competitive mating success for one transgenic C. hominivorax strain, CLAY (Allen et al. 2004a). Additional quality control assess-

Table 6. Combined comparison of strain traits, as proportions $\leq 1$ compared with P95 control

\begin{tabular}{|c|c|c|c|c|c|c|c|c|c|}
\hline Strain & P95 & CLAY & CLIX & CLOX & COTY & FOLY & GARY & GIZA & SUEZ \\
\hline Larval productivity (Table 4 , top) & 1 & 0.87 & 0.73 & 0.85 & 0.91 & 0.89 & 0.89 & 0.79 & 1.00 \\
\hline Adult emergence ${ }^{a}$ & 1 & 1.00 & 0.94 & 0.96 & 1.00 & 1.00 & 0.94 & 1.00 & 1.00 \\
\hline Male ratio ${ }^{1}$ & 1 & 1.00 & 1.00 & 1.00 & 1.00 & 0.99 & 1.00 & 1.00 & 0.99 \\
\hline Fecundity (Table 3) & 1 & 1.00 & 1.00 & 1.00 & 1.00 & 0.71 & 0.82 & 1.00 & 1.00 \\
\hline
\end{tabular}

Shading indicates a statistically significant fitness cost.

${ }^{a}$ Allen et al. (2004a). 
ments, such as starved longevity and flight ability (FAO/IAEA/USDA 2003) or other tests based on the type of insect and control application (van Lenteren et al. 2003) are appropriate for final (sterilized, if appropriate) production strains.

\section{Acknowledgments}

We thank Dennis Berkebile and technicians Brad Voelker, Nancy Lorenz, and Tony Weinhold (USDA-ARS-MLIRU Biosecure Rearing Facility) for excellent insect handling assistance. Linda J. Young provided essential statistical analysis guidance, and Muhammad Chaudhury provided helpful advice concerning the longevity studies. Livy Williams, Jian Chen, and Jarrod Leland reviewed and provided commentary on the manuscript.

\section{References Cited}

Allen, M. L., D. R. Berkebile, and S. R. Skoda. 2004a. Postlarval fitness of transgenic strains of Cochliomyia hominivorax (Diptera: Calliphoridae). J. Econ. Entomol. 97: 1181-1185.

Allen, M. L., A. M. Handler, D. R. Berkebile, and S. R. Skoda. 2004b. piggyBac transformation of the New World screwworm, Cochliomyia hominivorax, produces multiple distinct mutant strains. Med. Vet. Entomol. 18: 1-9.

Catteruccia, F., H. C. Godfray, and A. Crisanti. 2003. Impact of genetic manipulation on the fitness of Anopheles stephensi mosquitoes. Science (Wash. DC) 299: 1225-1227.

Chaudhury, M. F., L. A. Alvarez, and L. Lopez Velazquez. 2000. A new meatless diet for adult screwworm (Diptera: Calliphoridae). J. Econ. Entomol. 93: 1398-1401.
[FAO/IAEA/USDA]. Food and Agriculture Organization/ U.S. Department of Agriculture/International Atomic Energy Agency. 2003. Manual for product quality control and shipping procedures for sterile mass-reared tephritid fruit flies. International Atomic Energy Agency, Vienna, Austria.

Handler, A. M. 2001. A current perspective on insect gene transformation. Insect Biochem. Mol. Biol. 31: 111-128.

Handler, A. M. 2002. Prospects for using genetic transformation for improved SIT and new biocontrol methods. Genetica 116: 137-149.

Knipling, E. F. 1955. Possibilities of insect control or eradication through the use of sexually sterile males. J. Econ. Entomol. 48: 459-462.

Melvin, R., and R. C. Bushland. 1936. The nutritional requirements of screwworm larvae. J. Econ. Entomol. 33: $850-852$.

SAS Institute. 1999. SAS OnlineDoc, version 8.01. SAS Institute, Cary, NC.

Taylor, D. B., J. C. Bruce, and R. Garcia. 1991. Gelled diet for screwworm (Diptera: Calliphoridae) mass production. J. Econ. Entomol. 84: 927-935.

van Lenteren, J. C., A. Hale, J. N. Klapwijk, J. vanSchelt, and S. Steinberg. 2003. Guidelines for quality control of commercially produced natural enemies, pp. 265-303. In J. C. van Lenteren [ed.], Quality control and production of biological control agents: theory and testing procedures. CABI Publishing, Cambridge, MA.

Wyss, J. H. 2000. Screwworm eradication in the Americas. Ann. N.Y. Acad. Sci. 916: 186-193.

Received 20 April 2005; accepted 14 July 2005. 